\title{
Fast determination of capsaicinoids from peppers by high-performance liquid chromatography using a reversed phase monolithic column
}

\author{
G.F. Barbero, A. Liazid, M. Palma *, C.G. Barroso \\ Departamento de Química Analítica, Facultad de Ciencias, Universidad de Cádiz, P.O. Box 40, 11510 Puerto Real, Cádiz, Spain
}

Received 25 February 2007; received in revised form 19 June 2007; accepted 23 June 2007

\begin{abstract}
This article reports the development of a rapid and reproducible method of HPLC with fluorescence detection for the determination and quantification of the main capsaicinoids (nordihydrocapsaicin, capsaicin, dihydrocapsaicin, homocapsaicin and homodihydro-capsaicin) present in hot peppers by employing a monolithic column. The type of column employed is a RP-18e (100 mm $\times 4.6 \mathrm{~mm}) \mathrm{mono}-$ lithic column. A gradient method was utilised for the chromatographic separation: solvent A: water $(0.1 \%$ acetic acid $)$ and solvent B: methanol $(0.1 \%$ acetic acid). A study was also made of the robustness of the method in respect of the conditions of temperature in the separation column $\left(15-40{ }^{\circ} \mathrm{C}\right)$, the solvent flowrate $\left(4-7 \mathrm{~mL} \mathrm{~min}^{-1}\right)$, the injection volume $(10-50 \mu \mathrm{L})$, and the percentage of methanol in the sample $(25-100 \%)$. The repeatability and reproducibility of the method showed relative standard deviations of less than $2 \%$. The robustness of the method was determined by utilising different injection volumes and different percentages of methanol in the extracts. The method developed has then been utilised for the quantification of the major capsaicinoids present in different varieties of hot peppers grown in Spain. The capsaicinoids have been separated in a time of less than 8 min.
\end{abstract}

(C) 2007 Elsevier Ltd. All rights reserved.

Keywords: Capsaicinoids; Peppers; Liquid chromatography; Monolithic column

\section{Introduction}

Hot peppers, valued for their sensory attributes of pungency, aroma and colour, are among the most popular food additives around the world. The fruit is very important commercially, since large quantities and diverse varieties are consumed. The consumption of hot peppers is due mainly to their very pungent flavour. The pungency is caused by capsaicinoids, and among the most abundant of these components are capsaicin (trans- 8 methyl- $N$-vanillyl-6-nonenamide) and dihydrocapsaicin (8 methyl- $N$ vanillylnonanamide), which are responsible for about $90 \%$ of the spiciness (Laskaridou-Monnerville, 1999). In addition to capsaicin and dihydrocapsaicin, many less

\footnotetext{
* Corresponding author. Tel.: +34 956016360; fax: +34 956016460.

E-mail address: miguel.palma@uca.es (M. Palma).
}

abundant capsaicinoids have been detected in Capsicum extracts, including nordihydrocapsaicin, norcapsaicin, homocapsaicin, homodihydrocapsaicin, nornorcapsaicin, nornordihydrocapsaicin, nonivamide, etc. (Constant \& Cordell, 1996, 1995). An accurate determination of the levels of various capsaicinoids has become important because of the increasing demand by consumers for spicy foods, and the increasing use in pharmaceuticals (Kaale, Van Schepdael, Roets, \& Hoogmartens, 2002).

In addition to being widely utilised to give a peppery flavour to meals, capsaicinoids are molecules that have various other properties and applications that make these compounds very interesting to study. Principally they are compounds with notable anti-mutagenic and anti-tumoral properties (Surh et al., 1995; Toth \& Gannett, 1992) they also present a high antioxidant power (Henderson \& Slickman, 1999; Murakami, Ito, Htay, Tsubouchi, \& Yoshino, 
2001) and are much used as topical analgesics for treating many painful diseases, such as post-herpetic neuralgia (Morris, Gibson, \& Helme, 1995).

Several different methods, including organoleptic methods (Scoville Organoleptic Test) (Weaver, Luker, \& Neale, 1984), spectrophotometry (Ramos, 1979), thin layer chromatography (Lee, Suzuki, Kobashi, Hasegawa, \& Iwai, 1976), high-performance liquid chromatography (HPLC) (Games \& Alcock, 1984; Contreras-Padilla \& Yahia, 1998), and gas chromatography (Thomas, Schreiber, \& Weisskopf, 1998) have been used for the quantification of capsaicinoids from peppers and oleoresins. In recent years, reversed-phase HPLC has become the method most frequently used for analysis of capsaicinoids because of its rapidity and reliability. HPLC methods with ultraviolet (UV) (Weaver \& Awde, 1986), fluorescence (Barbero, Palma, \& Barroso, 2006) and electrochemical (Kawada, Watanabe, Katsura, Takami, \& Iwai, 1985) detection have been assayed for determining capsaicinoids.

Cooper, Guzinski, and Fisher (1991) developed a reversed-phase HPLC method utilising a conventional C-18 column to separate the three major capsaicinoids present in hot peppers (capsaicin, dihydrocapsaicin and nordihydrocapsaicin). The mobile phase that they utilised in isocratic regime was $60: 40(\mathrm{v} / \mathrm{v})$ methanol/water at a flowrate of $1.5 \mathrm{~mL} \mathrm{~min}{ }^{-1}$, and they obtained the separation of these three capsaicinoids in $28 \mathrm{~min}$.

Krajewska and Power (1986) also developed a reversedphase HPLC method utilising a conventional C-18 column to separate the two major capsaicinoids: capsaicin and dihydrocapsaicin in Capsicum fruit extracts. These authors utilised as mobile phase a gradient regime of methanol and water at a flowrate changing from 0.9 to $1.8 \mathrm{~mL} \mathrm{~min}^{-1}$, obtaining the separation of these two capsaicinoids in a time of $16 \mathrm{~min}$.

Kozukue et al. (2005) have recently developed a method for separating the three major and five minor capsaicinoids (homocapsaicin-I, homocapsaicin-II, homodihydrocapsaicin-I, homodihydrocapsaicin-II and nonivamide) present in peppers by employing LC-MS. The separation of these eight capsaicinoids was performed in a time of $66 \mathrm{~min}$.

Nowadays, high speed and low cost of analysis is increasingly being demanded in many areas where HPLC is applied, including pharmaceutical and food analysis, in order to increase throughput and reduce costs (Samanidou, Ioannou, \& Papadoyannis, 2004; Wu et al., 2004). The rapid separation of samples is an analytical stage that requires high efficiency as well as speed, due to the complexity of sample matrix, and hence is particularly challenging to achieve (Nováková, Matysová, Solichová, Koupparis, \& Solich, 2004). Monolithic columns have been investigated as a possible tool for reducing separation time in reversed-phase HPLC without significantly sacrificing efficiency or resolution (Tanaka et al., 2002; Ishizuka, Minakuchi, Nakanishi, Soga, \& Tanaka, 1998; Ishizuka et al., 2002). Several reviews have been published demonstrating the speed and cost-effectiveness of using this type of column in the analysis of a great variety of compounds (Calleri et al., 2004; Castellari, Sartini, Fabiani, Arfelli, \& Amati, 2002; Gerber et al., 2004).

This study reports the development of a new method of HPLC analysis, rapid and reproducible, applied to the main capsaicinoid compounds: nordihydrocapsaicin, capsaicin, dihydrocapsaicin, homocapsaicin and homodihydrocapsaicin, present in the hot varieties of peppers, by employing a monolithic column.

\section{Experimental procedures}

\subsection{Chemical and reagents}

Methanol and acetic acid (HPLC-grade) were purchased from Merck (Darmstadt, Germany). Purified water was prepared using a Milli-Q (Millipore, Bedford, MA, USA) water purification system. Capsaicin $(97 \%)$ and dihydrocapsaicin $(90 \%)$ were obtained from Sigma Chemical Co (St. Louis, MO, USA).

\subsection{Plant material}

The extracts employed for the development of the chromatographic method of separation of capsaicinoids were obtained from the hot Cayenne Pepper (Capsicum frutescens). The peppers were peeled, and the peduncle and seeds were separated. Only the pericarp and the placenta of the pepper were studied. Both the pericarp and the placenta were triturated with a conventional beater, until a homogeneous sample was obtained for the analysis. The triturated sample obtained was preserved in the freezer at $-32{ }^{\circ} \mathrm{C}$ until its subsequent extraction.

\subsection{Extraction of capsaicinoids}

The extract of the hot Cayenne Pepper (Capsicum frutescens) was obtained by using pressurised fluids according to the method developed by Barbero, Palma, and Barroso (2006) for the extraction of capsaicinoids. The extracts were filtered through a $0.45 \mu \mathrm{m}$ nylon syringe filter (Millex-HN, Ireland) before chromatographic analysis.

\subsection{Identification of capsaicinoids by liquid chromatography coupled to mass spectrometry}

A Finnigan LCQTM-coupled LC-MS system (Termo Electron Co., San Jose, CA) was used for the HPLC-MS analyses of extracts. This equipment is fitted with a Spectra SYSTEM 2000 model gradient pump (Thermo Separation Products, Fremont, USA) and a mass detector (model LCQ), it consists of an electrospray interface and an ion trap mass analyzer. Xcalibur, version 1.2 was the software used for the control of the equipment, and the acquisition and treatment of data. The sample injection volume was $25 \mu \mathrm{L}$. Interface conditions: positive ionization, temperature of the capillary: $220{ }^{\circ} \mathrm{C}$, spray voltage: $20 \mathrm{kV}$, capillary voltage: $-5 \mathrm{~V}$, focus gas flow: 80 (arbitrary units) and 
auxiliary gas flow: 10 (arbitrary units). API-MS spectra were acquired in the $m / z$ range of $50-400$.

The chromatographic method used was a gradient elution, using acidified water $(0.1 \%$ acetic acid, solvent $\mathrm{A})$ and acidified methanol $(0.1 \%$ acetic acid, solvent $\mathrm{B})$, at a flow rate of $0.2 \mathrm{~mL} \mathrm{~min}^{-1}$. The gradient employed was as follows: $0 \mathrm{~min}, 0 \% \mathrm{~B} ; 1 \mathrm{~min}, 0 \% \mathrm{~B} ; 5 \mathrm{~min}, 30 \% \mathrm{~B} ; 8 \mathrm{~min}$, $50 \% \mathrm{~B} ; 16 \mathrm{~min}, 70 \% \mathrm{~B} ; 20 \mathrm{~min}, 70 \% \mathrm{~B} ; 28 \mathrm{~min}, 90 \% \mathrm{~B}$; $30 \mathrm{~min}, 90 \% \mathrm{~B} ; 32 \mathrm{~min}, 100 \% \mathrm{~B} ; 42 \mathrm{~min}, 100 \%$ B. A C- 18 column (Luna $5 \mu \mathrm{m}, 150 \times 3 \mathrm{~mm}$, Phenomenex) was used for the chromatographic separation.

The capsaicinoids identified in the extracts of peppers analysed are: nordihydrocapsaicin (n-DHC), capsaicin (C), dihydrocapsaicin (DHC), homocapsaicin (h-C) and homodihydrocapsaicin (h-DHC).

\subsection{HPLC analysis}

The HPLC-Fluorescence analysis was performed in a Dionex (Sunnyvale, CA, USA) chromatographic system, consisting of an automated sample injector (ASI-100), pump (P680), thermostated column compartment (TCC100), a photodiode array detector (PDA-100), a fluorescence detector (RF 2000), a universal chromatography interface (UCI-50) and Chromeleon 6.60 software. Capsaicinoids were separated using a Chromolith Performance RP-18e $(100 \mathrm{~mm} \times 4.6 \mathrm{~mm})$ monolithic column (Merck). The wavelengths employed for the detection were $278 \mathrm{~nm}$ (excitation) and $310 \mathrm{~nm}$ (emission).

Evaluation of efficiency was made on basis of retention time, peak width, capacity factor $\left(k^{\prime}\right)$, separation factor $(\alpha)$, resolution and peak asymmetry. Resolution and asymmetry were calculated using the European Pharmacopeia standard. Resolution of each compound has been calculated related to the following peak eluted in the chromatogram.

\subsection{Calibration}

Using the method developed calibration curves were prepared with for capsaicin and dihydrocapsaicin $(y=$ $112,901 x+187$ for capsaicin and $y=151,770 x+4589$ for dihydrocapsaicin), which are the two standards of capsaicinoids commercially available. Regression equations and correlation coefficient $\left(r^{2}\right)$ were calculated using Microsoft Excel XP software ( 0.9995 for both C and DHC). The limits of detection $\left(0.008 \mathrm{mg} \mathrm{L}^{-1}\right.$ for capsaicin and $0.011 \mathrm{mg} \mathrm{L}^{-1}$ for dihydrocapsaicin) and quantification $\left(0.028 \mathrm{mg} \mathrm{L}^{-1}\right.$ for capsaicin and $0.036 \mathrm{mg} \mathrm{L}^{-1}$ for dihydrocapsaicin) were calculated using the ALAMIN software (Campana, Rodriguez, Barrero, Ceba, \& Fernández, 1997).

\section{Results and discussion}

\subsection{Isocratic methods}

Initially various different isocratic methods for the separation of the main capsaicinoids present in the peppers were studied. Five isocratic methods were assayed, working with different percentages of solvent B, from $40 \%$ to $60 \%$. The temperature in the column was held constant at $30{ }^{\circ} \mathrm{C}$.

The separation obtained between the compounds analysed by employing method $3(50 \%$ solvent A, 50\% solvent B) was acceptable. The methods with the greatest power of elution (methods 4 and 5) did not allow the initial peaks to be resolved, while the methods with less power of elution involved much longer times of analysis (19.2 min for method 2 and more than 30 min for method 1).

This isocratic method were utilised as point of departure for the development of a gradient method that enables the duration of the analysis to be reduced even further than $10 \mathrm{~min}$, which was the time need in the shortest isocratic method.

\subsection{Gradient method optimization}

Employing the same solvents and working at a flowrate

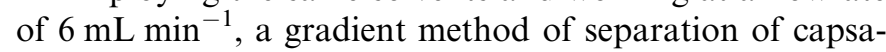
icinoids has been developed (time, solvent B): $0 \mathrm{~min}$, $10 \%$; $2 \mathrm{~min}, 50 \%$; $4 \mathrm{~min}, 50 \%$; $4.5 \mathrm{~min}, 55 \%$; $5.5 \mathrm{~min}$, $55 \%$; $6 \mathrm{~min}, 60 \%$; $7 \mathrm{~min}, 60 \%$; $9 \mathrm{~min}, 70 \%$; $10 \mathrm{~min}, 100 \%$; $15 \mathrm{~min}, 100 \%$. The temperature was held constant at $30^{\circ} \mathrm{C}$.

Employing this gradient method, a perfect separation of the capsaicinoids studied was obtained, with a good resolution of the chromatographic peaks, and notably shortening the time taken for the analysis, in comparison with the analysis time needed utilising C-18 columns in conventional reversed-phase HPLC (Cooper et al., 1991; Kozukue et al., 2005; Schweiggert, Carle, \& Schieber, 2006). The resulting retention times for the five capsaicinoids present in the hot Cayenne Pepper are shown in Fig. 1, in which the chromatogram obtained is presented. The analytical properties of the method developed, at a flowrate of $6 \mathrm{~mL} \min ^{-1}$. and at a column temperature of $30^{\circ} \mathrm{C}$, are presented in Table 1.

\subsection{Effect of temperature}

Having developed the gradient separation method, the effect of the temperature of the column on the chromatographic resolution of the peaks was evaluated. For this, the study was performed utilising several different column temperatures $\left(15,25,30,35\right.$ and $\left.40^{\circ} \mathrm{C}\right)$.

It was found that it is possible to achieve the separation of the capsaicinoids at temperatures between 30 and $35^{\circ} \mathrm{C}$. At $35^{\circ} \mathrm{C}$ the retention time of the last peak eluted was (7.12 $\mathrm{min})$, while at $30^{\circ} \mathrm{C}$ it was $(7.21 \mathrm{~min})$. However, the temperature of $30^{\circ} \mathrm{C}$ was selected since at this temperature the values of chromatographic resolution are higher (average resolution: 4.97 for $30^{\circ} \mathrm{C}$ and 3.95 for $35^{\circ} \mathrm{C}$ ). It was observed that if this temperature is increased or reduced, the chromatographic resolution of the peaks diminishes considerably, and at the temperature of $25^{\circ} \mathrm{C}$, dihydrocapsaicin was not separated from homocapsaicin, yet these 


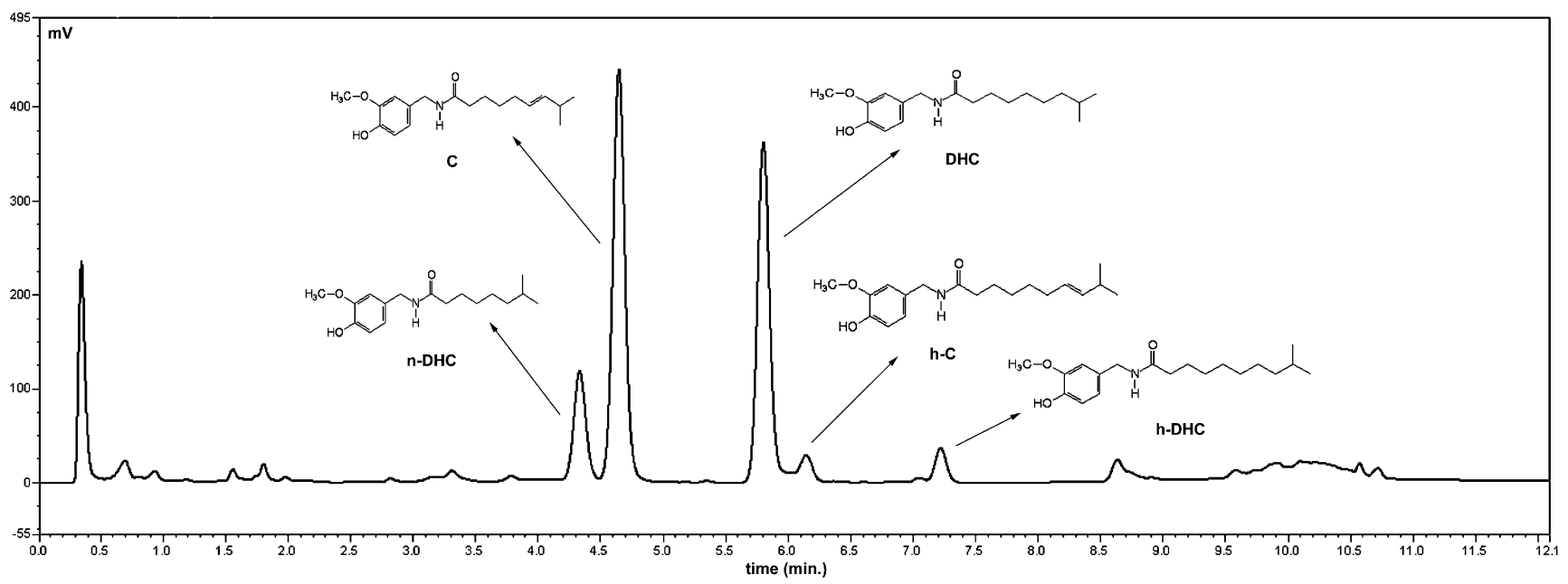

Fig. 1. Chromatogram of pepper extract and chemical structures of capsaicins (nordihydrocapsaicin (n-DHC), capsaicin (C), dihydrocapsaicin (DHC), homocapsaicin (h-C) and homodihydrocapsaicin (h-DHC)). Fluorescence detection: excitation $280 \mathrm{~nm}$, emission $310 \mathrm{~nm}$.

Table 1

Analytical properties (mean $\pm \mathrm{SD}(n=3)$ ) of the gradient method developed, at a flowrate of $6 \mathrm{~mL} / \mathrm{min}$ and at a column temperature of $30{ }^{\circ} \mathrm{C}$

\begin{tabular}{|c|c|c|c|c|c|}
\hline & n-DHC & $\mathrm{C}$ & DHC & h-C & h-DHC \\
\hline T. Ret. (min) & $4.29 \pm 0.05$ & $4.61 \pm 0.05$ & $5.78 \pm 0.04$ & $6.12 \pm 0.03$ & $7.21 \pm 0.01$ \\
\hline$k$ & $11.69 \pm 0.14$ & $12.61 \pm 0.15$ & $16.07 \pm 0.10$ & $17.08 \pm 0.09$ & $20.24 \pm 0.03$ \\
\hline Resolution & $1.77 \pm 0.02$ & $6.62 \pm 0.04$ & $1.86 \pm 0.01$ & $5.90 \pm 0.06$ & $8.16 \pm 0.14$ \\
\hline Asymmetry & $1.08 \pm 0.001$ & $1.04 \pm 0.005$ & $1.1 \pm 0.001$ & $1.03 \pm 0.001$ & $1.09 \pm 0.001$ \\
\hline Plates & $9587 \pm 148$ & $10914 \pm 932$ & $16793 \pm 335$ & $16145 \pm 1064$ & $28452 \pm 222$ \\
\hline
\end{tabular}

two compounds were perfectly separated at the temperature of $30^{\circ} \mathrm{C}$.

\subsection{Study of the effect of the flowrate of the mobile phase on the chromatographic separation}

The next parameter evaluated was the effect of the mobile phase flowrate on the separation of the majority capsaicinoids. The range of flowrates between 4 and $7 \mathrm{~mL} \mathrm{~min}^{-1}$ was studied.

Table 2 gives the resolutions and the retention times of the chromatographic peaks corresponding to the capsaicinoids studied at the various flowrates assayed. It can be observed that good chromatographic resolutions are achieved with solvent flowrates between 4 and $6 \mathrm{~mL} \mathrm{~min}^{-1}$. Above $6.5 \mathrm{~mL} \mathrm{~min}^{-1}$, the chromatographic resolutions begin to diminish, and at $7 \mathrm{~mL} \mathrm{~min}^{-1}$ dihydrocapsaicin is not chromatographically resolved from homocapsaicin.

However, the higher flowrates produce a significant shortening of the times of analysis: at $7 \mathrm{~mL} \mathrm{~min}^{-1}$ the capsaicinoids studied are eluted in only $6.2 \mathrm{~min}$, but this is at the cost of a worsening of the resolution of the chromatogram. Therefore the optimum flowrate for the separation of the five major capsaicinoids present in hot peppers is $6 \mathrm{~mL} \mathrm{~min}{ }^{-1}$. This flowrate gives an optimum resolution in the chromatographic separation of the peaks, with a notably reduced time of analysis $(7.21 \mathrm{~min})$. By this way the total time for the analysis of main capsaicinoids in peppers can be reduced dramatically from approx. $1.5 \mathrm{~h}$ per sample (as in Kozukue et al., 2005) to $0.25 \mathrm{~h}$ per sample (7.21 min plus cleaning and stabilization time for the next injection).

Table 2

Mean retention times in minutes $(n=3)$ and resolution $(n=3)$ of the chromatographic peaks corresponding to the capsaicinoids studied at each solvent flowrate assayed (n.a. not available, overlapped peaks)

\begin{tabular}{|c|c|c|c|c|c|c|c|c|c|c|}
\hline \multirow[t]{2}{*}{ Flowrate $\left(\mathrm{mL} \mathrm{min}{ }^{-1}\right)$} & \multicolumn{2}{|c|}{ n-DHC } & \multicolumn{2}{|l|}{$\mathrm{C}$} & \multicolumn{2}{|c|}{$\mathrm{DHC}$} & \multicolumn{2}{|l|}{$\mathrm{h}-\mathrm{C}$} & \multicolumn{2}{|c|}{ h-DHC } \\
\hline & $t$ & Res. & $t$ & Res. & $t$ & Res. & $t$ & Res. & $t$ & Res. \\
\hline 4.0 & 5.6 & 1.62 & 5.88 & 7.19 & 7.12 & 1.58 & 7.41 & 6.54 & 8.67 & 5.63 \\
\hline 5.0 & 4.93 & 1.58 & 5.17 & 8.3 & 6.42 & 1.55 & 6.69 & 6.88 & 7.93 & 7.82 \\
\hline 5.5 & 4.6 & 1.68 & 4.88 & 7.09 & 6.1 & 1.72 & 6.42 & 6.31 & 7.58 & 7.69 \\
\hline 6.0 & 4.29 & 1.77 & 4.61 & 6.62 & 5.78 & 1.86 & 6.12 & 5.90 & 7.21 & 8.72 \\
\hline 6.5 & 4.17 & 1.68 & 4.45 & 6.27 & 5.5 & 1.69 & 5.8 & 5.84 & 6.86 & 8.11 \\
\hline 7.0 & 3.74 & 1.47 & 3.94 & 7.58 & 4.95 & n.a. & 5.14 & n.a. & 6.2 & 6.14 \\
\hline
\end{tabular}




\subsection{Repeatability and reproducibility of the method}

The repeatability and reproducibility of the method developed was studied in respect of the peak area of each capsaicinoid, the chromatographic resolution and the capacity factor. This involved performing a total of 24 analyses of the same sample (extract of hot Cayenne Pepper) distributed as follows: 12 analyses performed on the first day of the study and six more analyses on each of the two consecutive days.

The average (five capsaicinoides) relative standard deviations intraday and interday in respect of the peak area were $0.73 \%$ and $1.72 \%$, respectively. For chromatographic resolution of the peaks, the resulting deviations intraday and interday were $1.35 \%$ and $1.70 \%$ respectively. Finally for the capacity factor the average relative standard deviation for intraday analyses was $0.39 \%$ whereas for interday analyses the result was $0.72 \%$.

It can be confirmed that in all the cases, the RSD are below $2 \%$, for the area, the resolution and the capacity factor, which shows that this is a method with high reproducibility of results.

\subsection{Robustness of the method}

\subsubsection{Injection volume}

To determine the robustness of the method in respect of the injection volume, analyses were performed employing different injection volumes (10, 20, 30, 40 and $50 \mu \mathrm{L})$, using an extract of the hot Cayenne Pepper. The effect of this variable on three parameters: capacity factor of the chromatographic peaks, chromatographic resolutions of the peaks, and areas of the chromatographic peaks, was checked. Each assay was performed in triplicate.

No significant differences $(p<0.05)$ were found between the values of the capacity factors for the different peaks, from which it can be concluded that the capacity factors are not influenced by varying the injection volume in the range between 10 and $50 \mu \mathrm{L}$.

With respect to the influence on the peak area by modifying the injection volumes $(10-50 \mu \mathrm{L})$, regression curves were drawn for each of the capsaicinoids studied, so as to check the linearity of the curves. The highest RSD found for the slopes of the curves was 2.71. Therefore the calibration curves show a good linearity when the injection volumes are modified, i.e. in respect of the areas of the chromatographic peaks, this method is clearly robust against variations in the injection volumes.

With respect to the influence on the chromatographic resolution, an analysis of the variance of a factor has been performed. The data obtained are given in Table 3. It can be observed that there are no statistically significant differences with respect to the resolution of the chromatographic peaks, from injection volumes ranging between 10 and $30 \mu \mathrm{L}$. Above $40 \mu \mathrm{L}$ it is observed that the resolution values begin to be significantly different in the case of capsaicin, dihydrocapsaicin, homocapsaicin and homodihydrocapsaicin. This
Table 3

Analysis of the variance of a factor for the resolution, when the injection volumes are modified between 10 and $50 \mu \mathrm{L}$

\begin{tabular}{llllll}
\hline Volume $(\mu \mathrm{L})$ & n-DHC & C & DHC & h-C & h-DHC \\
\hline 10 & $1.73^{\mathrm{a}}$ & $6.72^{\mathrm{a}}$ & $1.84^{\mathrm{a}}$ & $5.92^{\mathrm{a}}$ & $8.54^{\mathrm{a} . \mathrm{b}}$ \\
20 & $1.75^{\mathrm{a}}$ & $6.73^{\mathrm{a}}$ & $1.77^{\mathrm{a}, \mathrm{b}}$ & $5.84^{\mathrm{a}}$ & $8.71^{\mathrm{a} . \mathrm{b}}$ \\
30 & $1.76^{\mathrm{a}}$ & $6.62^{\mathrm{a}}$ & $1.8^{\mathrm{a}, \mathrm{b}}$ & $5.79^{\mathrm{a}}$ & $8.83^{\mathrm{a}}$ \\
40 & $1.70^{\mathrm{a}}$ & $6.36^{\mathrm{b}}$ & $1.72^{\mathrm{b}}$ & $5.54^{\mathrm{b}}$ & $8.41^{\mathrm{b}}$ \\
50 & $1.54^{\mathrm{b}}$ & $6.16^{\mathrm{c}}$ & $1.62^{\mathrm{c}}$ & $5.27^{\mathrm{c}}$ & $8.37^{\mathrm{b}}$ \\
\hline
\end{tabular}

Means with the same superscripts $(\mathrm{a}, \mathrm{b}, \mathrm{c})$ in the same column are not statistically different $(P<0.05)$.

finding means that the injection volume for the method developed should be limited to $30 \mu \mathrm{L}$ for the values of concentration of the capsaicinoids employed in these analyses.

\subsubsection{Sample solvent}

When working with samples who's content in water is very high, the extraction methods that are employed provide extracts with different proportions of organic solvent in water. The presence of the organic solvent can cause distortions in the chromatographic peaks, therefore it is necessary to evaluate the effect of the sample solvent on the chromatographic peaks obtained. For this, extracts with different percentages of methanol $(25 \%, 50 \%, 75 \%$ and $100 \%$ ) in water were employed. The effect of the percentage of methanol on the peak area, the capacity factor and the chromatographic resolution was studied. This required an analysis of the variance of a factor for each of the parameters to be studied. The volume of extract injected was $25 \mu \mathrm{L}$. Each analysis was performed in triplicate.

No statistically significant differences were observed in respect of the capacity factors of the peaks nor in respect of the chromatographic resolutions of the peaks; it was therefore concluded that these two parameters are not influenced by the percentage of methanol that may be present in the extract.

However significant differences were observed with the areas of the peaks when the percentage of methanol in the extracts was varied, as indicated in Table 4. It can be confirmed that the values of the areas corresponding to extracts containing $100 \%$ methanol are significantly different from the rest.

Therefore, the method developed presents a high degree of robustness with respect to the capacity factor and to the resolution, when the percentage of methanol in the extracts

Table 4

Areas corresponding to the different percentages of methanol $(25-100 \%)$ in the extracts

\begin{tabular}{llllll}
\hline$\% \mathrm{MeOH}$ & $\mathrm{n}-\mathrm{DHC}$ & $\mathrm{C}$ & $\mathrm{DHC}$ & $\mathrm{h}-\mathrm{C}$ & $\mathrm{h}-\mathrm{DHC}$ \\
\hline 25 & $16,883^{\mathrm{a}}$ & $65,076^{\mathrm{a}}$ & $55,932^{\mathrm{a}}$ & $4916^{\mathrm{a}}$ & $5323^{\mathrm{a}}$ \\
50 & $17,096^{\mathrm{a}}$ & $65,218^{\mathrm{a}}$ & $55,919^{\mathrm{a}}$ & $4984^{\mathrm{a}}$ & $5370^{\mathrm{a}}$ \\
75 & $17,224^{\mathrm{a}}$ & $65,626^{\mathrm{a}}$ & $56,043^{\mathrm{a}}$ & $4970^{\mathrm{a}}$ & $5458^{\mathrm{a}}$ \\
100 & $14,750^{\mathrm{b}}$ & $56,697^{\mathrm{b}}$ & $48,142^{\mathrm{b}}$ & $4210^{\mathrm{b}}$ & $4708^{\mathrm{b}}$ \\
\hline
\end{tabular}

Means with the same superscripts $(a, b)$ in the same column are not statistically different $(P<0.05)$. 
Table 5

Quantity $(\mu \mathrm{mol})$ of capsaicinoid per kilogram of fresh pepper in the samples analysed $(n=3)$

\begin{tabular}{|c|c|c|c|c|c|}
\hline Sample & n-DHC & $\mathrm{C}$ & $\mathrm{DHC}$ & h-C & h-DHC \\
\hline Var. 1 & $141.2 \pm 3.4$ & $579.0 \pm 14.2$ & $528.7 \pm 13.5$ & $22.5 \pm 0.5$ & $43.4 \pm 1.1$ \\
\hline Var. 2 & $271.7 \pm 6.6$ & $800.9 \pm 19.7$ & $857.8 \pm 20.0$ & $78.2 \pm 1.9$ & $95.6 \pm 2.5$ \\
\hline Var. 3 & $13.3 \pm 0.3$ & $60.1 \pm 1.8$ & $52.6 \pm 1.3$ & n.d. & $3.5 \pm 0.1$ \\
\hline Var. 4 & $55.2 \pm 1.3$ & $282.5 \pm 6.9$ & $195.4 \pm 5.0$ & $9.9 \pm 0.2$ & $19.8 \pm 0.5$ \\
\hline Var. 5 & $24.6 \pm 0.6$ & $158.9 \pm 3.9$ & $95.4 \pm 2.4$ & $10.8 \pm 0.3$ & $10.9 \pm 0.3$ \\
\hline Var. 6 & $451.4 \pm 11.0$ & $1151.8 \pm 28.3$ & $1404.0 \pm 35.9$ & $71.3 \pm 1.7$ & $156.1 \pm 4.0$ \\
\hline Var. 7 & $30.6 \pm 0.7$ & $130.2 \pm 3.2$ & $88.7 \pm 2.3$ & $5.4 \pm 0.1$ & $13.2 \pm 0.3$ \\
\hline Var. 8 & $330.0 \pm 8.0$ & $974.6 \pm 23.9$ & $1070.7 \pm 27.4$ & $34.5 \pm 0.8$ & $52.4 \pm 1.4$ \\
\hline
\end{tabular}

is modified, but the robustness of the method is lower in respect of the area of the chromatographic peaks. This means that, in the case of samples whose percentage of methanol exceeds $75 \%$, it is necessary first to eliminate part of the methanol or else to dilute with water, in order to be able to utilise the method developed.

\subsection{Quantification of the capsaicinoids present in different varieties of hot chili peppers cultivated in Spain}

Having developed the optimum method for the analysis of capsaicinoids by employing a monolithic column, a further study was carried out to quantify the major capsaicinoids present in eight varieties of hot chili peppers cultivated in Spain. The results are shown in Table 5.

The variety with the greatest content in capsaicinoids was var. 6. It can be observed that, in general, capsaicin is the major capsaicinoid in most of the hot varieties of peppers (Table 5); however, in certain cases like the varieties 2, 6 and 8, which are also the most pungent of the varieties studied; the major capsaicinoid is dihydrocapsaicin (Table 5).

\section{Conclusions}

This study reports the development of a rapid and reproducible method for the separation of capsaicinoids using a monolithic column. A notable shortening of the time of analysis $(7.22 \mathrm{~min})$ has been achieved for the five major capsaicinoids (nordihydrocapsaicin, capsaicin, dihydrocapsaicin, homocapsaicin and homodihydrocapsaicin) present in hot peppers, in comparison with the existing methods described in the bibliography that utilise a conventional C-18 column in reversed-phase HPLC.

The method developed utilises a working temperature of $30{ }^{\circ} \mathrm{C}$ in the column and a flowrate of $6 \mathrm{~mL} \mathrm{~min}{ }^{-1}$. With these conditions a rapid method is achieved for the separation and analysis of these capsaicinoids, with a high repeatability and reproducibility (RSD $<2 \%$ ) both for the peak area and for the capacity factor of the capsaicinoids and for the resolution of the chromatographic peaks.

The method developed has been shown to be robust with respect to the area of the peaks and to their capacity factor by modifying the sample injection volumes; however the method is only robust for injection volumes of up to
$30 \mu \mathrm{L}$ if the resolutions are considered. It was also found to be robust with respect to the capacity factor and resolution of the chromatographic peaks, by modifying the percentage of methanol in the extracts. However significant differences were found when the composition of the solvent in which the sample is dissolved is $100 \%$ methanol, if the peak areas are considered.

\section{Acknowledgements}

G.F. Barbero gratefully thanks the Ministerio de Educación y Ciencia for a doctoral scholarship. This study was supported by the CICYT (Project BIO2000-1091-C02-02).

\section{References}

Laskaridou-Monnerville, A. (1999). Journal of Chromatography A, 838, 293. Constant, H. L., \& Cordell, G. A. (1996). Journal of Natural Products, 59, 425.

Constant, H. L., \& Cordell, G. A. (1995). Journal of Natural Products, 58, 1925.

Kaale, E., Van Schepdael, A., Roets, E., \& Hoogmartens, J. (2002). Journal of Pharmaceutical and Biomedical Analysis, 30, 1331.

Toth, B., \& Gannett, P. (1992). In vivo, 6, 59.

Surh, Y. J., Lee, C. R. J., Park, K. K., Mayne, S. T., Liem, A., \& Miller, J. A. (1995). Carcinogenesis, 16, 2467.

Henderson, D. E., \& Slickman, A. M. (1999). Journal of Agriculture and Food Chemistry, 47, 2563.

Murakami, K., Ito, M., Htay, H. H., Tsubouchi, R., \& Yoshino, M. (2001). Biomedical Research-Tokyo, 22, 15

Morris, G. C., Gibson, S. J., \& Helme, R. D. (1995). Pain, 63, 93.

Weaver, K. M., Luker, R. G., \& Neale, M. E. (1984). Journal of Chromatography, 301, 288.

Ramos, P. J. J. (1979). Journal - Association of Official Analytical Chemists, 62, 1168.

Lee, K. R., Suzuki, T., Kobashi, M., Hasegawa, K., \& Iwai, K. (1976). Journal of Chromatography, 123, 119.

Games, D. E., \& Alcock, N. J. (1984). Journal of Chromatography A, 294, 269.

Contreras-Padilla, M., \& Yahia, E. M. (1998). Journal of Agriculture and Food Chemistry, 46, 2075.

Thomas, B. V., Schreiber, A. A., \& Weisskopf, C. P. (1998). Journal of Agriculture and Food Chemistry, 46, 2655.

Weaver, K. M., \& Awde, D. B. (1986). Journal of Chromatography A, 367, 438.

Barbero, G. F., Palma, M., \& Barroso, G. C. (2006). Analytica Chimica Acta, 578, 227.

Kawada, T., Watanabe, T., Katsura, K., Takami, H., \& Iwai, K. (1985). Journal of Chromatography A, 329, 99.

Cooper, T. H., Guzinski, J. A., \& Fisher, C. (1991). Journal of Agriculture and Food Chemistry, 39, 2253. 
Krajewska, A. M., \& Power, J. J. (1986). Journal of Chromatography A, $367,267$.

Kozukue, N., Han, J. S., Kozukue, E., Lee, S. J., Kim, J. A., Lee, K. R., et al. (2005). Journal of Agriculture and Food Chemistry, 53, 9172.

Samanidou, V. F., Ioannou, A. S., \& Papadoyannis, I. N. (2004). Journal of Chromatography B, 809, 175.

Wu, N., Dempsey, J., Yehl, P. M., Dovletoglou, A., Ellison, D., \& Wyvratt, J. (2004). Analytica Chimica Acta, 523, 149.

Nováková, L., Matysová, L., Solichová, D., Koupparis, M. A., \& Solich, P. (2004). Journal of Chromatography B, 813, 191.

Ishizuka, N., Minakuchi, H., Nakanishi, K., Soga, N., \& Tanaka, N. (1998). Journal of Chromatography A, 797, 133.

Tanaka, N., Kobayashi, H., Ishizuka, N., Minakuchi, H., Nakanishi, K., \& Ikegami, T. (2002). Journal of Chromatography A, 965, 35.
Ishizuka, N., Kobayashi, H., Minakuchi, H., Nakanishi, K., Hirao, K., Hosoya, K., et al. (2002). Journal of Chromatography A, 960, 85.

Gerber, F., Krummen, M., Potgeter, H., Roth, A., Siffrin, C., \& Spoendlin, C. (2004). Journal of Chromatography A, 1036, 127.

Calleri, E., Massolini, G., Lubda, D., Temporini, C., Loiodice, F., \& Caccialanza, G. (2004). Journal of Chromatography A, 1031, 93.

Castellari, M., Sartini, E., Fabiani, A., Arfelli, G., \& Amati, A. (2002). Journal of Chromatography A, 973, 221.

Barbero, G. F., Palma, M., \& Barroso, G. C. (2006). Journal of Agriculture and Food Chemistry, 54, 3231.

Campana, A. M. G., Rodriguez, L. C., Barrero, F. A., Ceba, M. R., \& Fernández, J. L. S. (1997). Trends in Analytical Chemistry, 16, 381.

Schweiggert, U., Carle, R., \& Schieber, A. (2006). Analytica Chimica Acta, $557,236$. 